\title{
Vulnerability of People with Cancer and the Potential Risks of COVID-19 Pandemic: A perspective in Morocco
}

\author{
Rachid Ait Addi ${ }^{1, *}$, Abdelhafid Benksim ${ }^{1,2}$, Mohamed Cherkaoui ${ }^{2}$
}

\author{
${ }^{1}$ Laboratory of Human Ecology, \\ Department of Biology, School of \\ Sciences Semlalia, Cadi Ayyad University, \\ Marrakech. Morocco \\ ${ }^{2}$ High Institute of Nursing and Technical \\ Health, Marrakech, Morocco
}

*Correspondence

dr.rachid.aitaddi@gmail.com

(Rachid Ait Addi)

\begin{abstract}
Cancer affects almost 18 million around the world in 2018, of these 9.5 million cases were in men and 8.5 million in women. COVID-19 is an acute respiratory disease caused by novel coronavirus SARS-CoV-2 or 2019-nCoV and was first reported in Wuhan, China in December 2019. Recently, on March 11, 2020, COVID-19 was declared by the World Health Organization (WHO) as a virus pandemic disease. Furthermore, it has been known that the malignancy and anticancer treatments, such as chemotherapy or surgery cause systemic immunosuppressive state in patients with cancer which exposes them to infection more than others especially for COVID-19 and with a high probability of complications. Additionally, the major concern for patients with cancer is the incapacity to get fundamental medical services due to the pandemic spread. In Morocco, thanks to measures such as contamination prevention, prioritization of care, and price control of therapies, cancer patients were able to continue their treatment during the covid-19 pandemic.
\end{abstract}

\section{Keywords}

COVID-19, Cancer, Pandemic, Morocco
In 2018, the incidence of cancer around the world was almost 18 million, of these 9.5 million cases were in men and 8.5 million in women [1]. This number is growing because of the multiple risk factors such as age, tobacco, alcohol, diet, radiation, obesity, cancer-causing substances, chronic Inflammation, infectious agents, and sunlight [2].

In Morocco, the Age-specific rate (ASR) is 31.9 per 100,000 persons in 2018 [3]. This fragile population needs a specific care such as surgery, chemotherapy, radiation therapy, immunotherapy, hormone therapy, and psychological support [4].

COVID-19 is an acute respiratory disease caused by novel coronavirus SARS-CoV-2 or 2019-nCoV which was first reported in Wuhan, China in December 2019. Recently, on March 11, 2020, COVID-19 was declared by the World Health Organization (WHO) as a virus pandemic disease [5-8].

Many risk factors are recognized to be related to illness from COVID-19. Age over 65 years old, respiratory conditions, diabetes, serious heart conditions, chronic kidney disease being treated with dialysis, severe obesity, people in nursing homes or long-term care facilities, immunocompromised, liver disease, and cancer [9, 10].
Also, the malignancy and anticancer treatments, such as chemotherapy or surgery cause systemic immunosuppressive state in patients with cancer. This is the reason why cancer patients are more vulnerable to infection than others and subsequently they are at high risk for COVID19 infection and its complications [11, 12].

Additionally, severe events (admission to the intensive care unit and requiring invasive ventilation, or death) are most frequent amongst patients with cancer and whom had chemotherapy or surgery in the past month than respectively patients without cancer and whom did not have chemotherapy or surgery [11].

Likewise, in this COVID-19 pandemic, the major concern for patients with cancer is the incapacity to get fundamental medical services due to the pandemic spread. In addition, patients are recommended to avoid visiting hospitals because of the risk of infection. As a result, some clinical trials are delayed which complicates frequentation of the hospital for regular appointments or continuity of care, and treatment delays or unavailability may cause possible concerns in case of severe complications or emergencies in patients with advanced cancers [12].

Likewise, the diversion of attention exclusively to 
COVID-19 can have disastrous consequences especially for patients with cancer. The excessive deployment of medical and paramedical staff to the COVID-19 service can leave certain potentially vital activities uncovered, such as the administration of treatment, surgeries and assistance to hospitalized patients. Otherwise, it is well documented that delayed oncologic surgery can cause disease progressions and result in tumors no longer resectable, leading to worse survival outcomes [13].

In fact, immediate diagnosis and treatment are needed in several solid tumors and hematologic cancers [14, 15]. Moreover, for patients with head and neck cancer, the risk of death increased at $16 \%$ for every month of delay of radiotherapy [16]. Also, inferior survival was related to delays in receiving adjuvant chemotherapy for colorectal cancer and breast cancer [16]. Furthermore, worse survival outcomes were associated to four weeks of delay before the initiation of adjuvant chemotherapy in gastric cancer patients [17].

In Morocco, the AMOT (Moroccan Association of Thoracic Oncology), recommends three essential points, namely prevention of contamination, prioritization of care, and price control of therapies of patients by the various oncology centers and services. In fact, to avoid contamination of patients, AMOT recommends increased vigilance through the temperature measurement and the search for respiratory and digestive symptoms on a regular basis, whether for patients or for nursing and administrative staff. Also, minimizing frequency of hospital visits, respect of the measures of distancing, wearing Personal Protective Equipment (PPE), and use of rigorous hand hygiene practices were highly recommended.

Moreover, we note that these recommendations, were shared with nearly 400 cancer specialists, and should allow patients to continue the most optimal treatments, avoiding the risk of contamination with Covid-19 as much as possible [18].

Fortunately, most of cancer patients continue their treatment during the COVID-19 pandemic in Morocco. This will allow healing to progress and will avoid relapses as much as possible.

Finally, vigilance must continue as well as all preventive measures to avoid contamination of cancer patients with COVID-19, and more awareness programs for this category of patients must be conducted in order to educate them on the risks they run as well as the means to prevent contamination.

\section{ACKNOWLEDGMENTS}

We would like to express our gratitude to all those who helped us during the writing of this manuscript. We also thank all the peer reviewers and editors for their opinions and suggestions. Finally, authors declare no funding support.

\section{CONFLICTS OF INTEREST}

The authors declare no conflict of interest.

\section{REFERENCES}

[1] Worldwide cancer data Global. Cancer statistics for the most common cancers. https://www.wcrf.org/dietandcancer/cancertrends/worldwide-cancer-data.

[2] National Cancer Institute. Risk Factors for Cancer. https ://www . cancer.gov/about-cancer/causes-prevention/risk.

[3] Bray F, Ferlay J, Soerjomataram I, Siegel RL, Torre LA, and Jemal A. Global cancer statistics 2018: GLOBOCAN estimates of incidence and mortality worldwide for 36 cancers in 185 countries. CA Cancer J Clin. 2018;68:394-424.

[4] National Cancer Institute.Cancer Treatment. https://www. cancer.gov/about-cancer/treatment.

[5] Ait Addi R, Benksim A, Amine M, Cherkaoui M. COVID19 Outbreak and Perspective in Morocco. Electron J Gen Med. 2020;17:em204.

[6] Gao Y, Li T, Han M, et al. Diagnostic utility of clinical laboratory data determinations for patients with the severe COVID-19. J Med Virol. 2020;1-6.

[7] Bai Y, Yao L, Wei T, et al. Presumed Asymptomatic Carrier Transmission of COVID-19. JAMA. 2020;323:1406-1407.

[8] Kruse RL. Therapeutic strategies in an outbreak scenario to treat the novel coronavirus originating in Wuhan,China. 1000Res. 2020;9:72.

[9] Centers for disease control and prevention. Coronavirus Disease 2019 (COVID-19) : People Who Are At Higher Risk. https ://www.cdc.gov/coronavirus/2019-ncov/needextra-precautions/groups-at-higher-risk.html.

[10] Lambertini M, Toss A, Passaro A, et al. Cancer care during the spread of coronavirus disease 2019 (COVID-19) in Italy: young oncologists' perspective. ESMO Open. 2020;5:e000759.

[11] Hanping Wang, Li Zhang. Risk of COVID-19 for patients with cancer. Lancet oncol. 2020;21:E181.

[12] Liang W, Guan W, Chen R, Wang W, Li J, Xu K, Li C, Ai Q, Lu W, Liang H, Li S, He J. Cancer patients in SARS-CoV-2 infection: a nationwide analysis in China. Lancet Oncol. 2020;21:335-337.

[13] Cortiula F, Pettke A, Bartoletti M, Puglisi F, Helleday T. Managing COVID-19 in the oncology clinic and avoiding the distraction effect. Ann Oncol. 2020;31:553-555.

[14] Rivera A, Ohri N, Thomas E, Miller R, Knoll MA. The Impact of COVID-19 on Radiation Oncology Clinics and Cancer Patients in the U.S. Adv Radiat Oncol. 2020. doi:10.1016/j.adro.2020.03.006.

[15] Yuen E, Fote G, Horwich P, et al. Head and neck cancer care in the COVID-19 pandemic: A brief update. Oral Oncol. 2020;105:104738.

[16] Hanna TP, Evans GA, Booth CM. Cancer, COVID-19 and the precautionary principle: prioritizing treatment during a global. Nat Rev Clin Oncol. 2020;17:268-270.

[17] Lu H, Zhao B, Zhang J, et al. Does delayed initiation of adjuvant chemotherapy following the curative resection affect the survival outcome of gastric cancer patients: A systematic review and metaanalysis [published online ahead of print, 2020 Jan 8]. Eur J Surg Oncol. 2020;S0748-7983:30017-2.

[18] Covid-19/Maroc : Ne pas oublier les patients atteints de cancer $\mathrm{du}$ poumon. https://fr.hespress.com/142811-covid-19maroc-ne-pas-oublier-les-patients-\atteints-decancer-du-poumon.html.

How to cite this article: Rachid Ait Addi, Abdelhafid Benksim, Mohamed Cherkaoui. Vulnerability of People with Cancer and the Potential Risks of COVID-19 Pandemic: A perspective in Morocco. Signa Vitae.2020;16(1):207-208. doi:10.22514/sv.2020.16.0030. 